\title{
Effect of icariin on cell proliferation and the expression of bone resorption/formation-related markers in human periodontal ligament cells
}

\author{
ZHENHUA PEI $^{1,2}$, FENGQIU ZHANG ${ }^{1}$, ZHONGYING NIU $^{2}$ and SHENGGEN SHI ${ }^{2}$ \\ ${ }^{1}$ Department of Periodontics, Capital Medical University, School of Stomatology, Beijing 100050; \\ ${ }^{2}$ Department of Stomatology, The 306th Hospital of PLA, Beijing 100101, P.R. China
}

Received March 28, 2013; Accepted August 12, 2013

DOI: $10.3892 / \mathrm{mmr} .2013 .1696$

\begin{abstract}
Periodontitis is a common destructive inflammatory disease that leads to changes in the tooth-supporting tissues. Human periodontal ligament cells are essential in periodontal tissue regeneration. The traditional Chinese medicine icariin promoted bone formation, stimulated the osteogenic differentiation of preosteoblastic cells and inhibited osteoclast differentiation and bone resorption. Thus, in the present study, the effect of icariin on cell proliferation and the expression of osteoprotegerin (OPG), receptor activator of nuclear factor- $\kappa \mathrm{B}$ ligand (RANKL), core binding factor $\alpha 1$ (Cbfa1) and osteocalcin (OC) was investigated in human periodontal ligament cells, by an MTT assay, qPCR and western blot analysis. The results demonstrated that icariin promoted cell proliferation in a dose- and time-dependent manner, upregulated OPG, Cbfa1 and OC expression, and downregulated RANKL production and the RANKL/OPG expression ratio. This suggested the potential value of icariin in treating alveolar bone resorption and promoting periodontal tissue regeneration, due to its ability to stimulate the proliferation and osteogenic differentiation of human periodontal ligament cells and inhibit osteoclast differentiation.
\end{abstract}

\section{Introduction}

Periodontitis is a common inflammatory disease that is characterized by the destruction of tooth-supporting tissues, including alveolar bone, and is the most common cause of tooth loss in adults (1). For decades, one of the key obstacles in periodontitis therapy is how to inhibit alveolar bone resorption

Correspondence to: Professor Fengqiu Zhang, Department of Periodontics, Capital Medical University, School of Stomatology, 4 Tiantan Xili, Fengtai, Beijing 100050, P.R. China

E-mail:niuzy306@gmail.com

Key words: icariin, human periodontal ligament cells, osteoprotegerin, receptor activator of nuclear factor- $\kappa \mathrm{B}$ ligand, core binding factor $\alpha 1$, osteocalcin and promote bone formation. The core mechanism of alveolar bone resorption in periodontitis is osteoclast activation $(2,3)$. Human periodontal ligament cells (HPLCs), the predominant cellular constituents of the periodontium, are important in maintaining the integrity of the tooth-supporting tissues in periodontitis. In addition to exhibiting multi-potential mesenchymal stromal cell characteristics, such as osteoblastic and cementoblastic differentiation potential $(4,5)$, HPLCs have been shown to affect osteoclastogenesis. Osteoprotegerin produced by periodontal ligament fibroblasts prevented pre-osteoclast differentiation and function (6-8), and the conditioned media of periodontal ligament fibroblasts inhibited osteoclast formation in a mouse bone marrow culture (6).

Icariin is the predominant active compound of the total flavonoid extracted from the stem and leaves of Epimedium species in traditional Chinese medicine. In addition, it has been demonstrated to stimulate the proliferation and osteogenic differentiation of human bone mesenchymal stem cells, promotes bone formation and inhibits cell apoptosis, osteoclast differentiation and bone resorption (9-12). Icariin is suggested to enhance bone healing and reduce the occurrence of osteoporosis and had been considered as a candidate osteogenic compound for use in bone tissue engineering (10,13-15).

With respect to the diverse pharmacological activities of icariin, in the present study, it was investigated whether icariin promoted HPLC proliferation and regulated the expression of biomarkers associated with bone formation or resorption, such as RANKL, OPG, Cbfa1 and OC in HPLCs. The results of the study thereby offer an insight into its use as a therapy for periodontitis and periodontal tissue regeneration.

\section{Materials and methods}

Reagents. Icariin was obtained from the National Institute for the Control of Pharmaceutical and Biological Products (NICPBP; Beijing, China) with a purity of 99\%. Stock solutions of icariin were prepared in dimethylsulfoxide (DMSO; Sigma-Aldrich, St. Louis, MO, USA) and stored at $-20^{\circ} \mathrm{C}$. The final concentrations of icariin used in the culture were 0.001 , $0.01,0.1$ and $1 \mu \mathrm{g} / \mathrm{ml}$. Dulbecco's modified Eagle's medium (DMEM), trypsin and TRIzol reagent were purchased from Gibco-BRL (St. Louis, MO, USA); fetal bovine serum (FBS) 
Table I. Oligonucleotide primers used for qPCR.

\begin{tabular}{llr}
\hline Primer & \multicolumn{1}{c}{ Sequence } & Amplicon size (bp) \\
\hline$\beta$-actin & Forward: 5'-TGACGTGGACATCCGCAAAG-3' & 205 \\
& Reverse: 5'-CTGGAAGGTGGACAGCGAGG-3' & \\
RANKL & Forward: 5'-TGGATGGCTCATGGTTAGAT-3' & 160 \\
OPG & Reverse: 5'-GTCATGTTGGAGATCTTGGC-3' & 211 \\
Cbfa1 & Forward: 5'-GAAAGTGGGAGCAGAAGACA-3' \\
OC & Reverse: 5'-GAAGCTGTGAAGGAACCTGA-3' & 122 \\
& Forward: 5'-CTATCAGTTTCCCATGGTGC-3' & 148 \\
\hline
\end{tabular}

RANKL, receptor activator of nuclear factor- $\kappa \mathrm{B}$ ligand; OPG, osteoprotegerin; $\mathrm{CbFa1}$, core binding factor $\alpha 1$; OC, osteocalcin.

was purchased from the Thermo Scientific HyClone (South Logan, UT, USA); polyclonal rabbit anti-osteoprotegerin (OPG) and polyclonal rabbit anti-receptor activator of nuclear factor- $\kappa$ B ligand (RANKL) antibodies were obtained from Santa Cruz Biotechnology (Santa Cruz, Santa Cruz, CA, USA); polyclonal rabbit anti-core binding factor $\alpha 1$ (Cbfa1) and polyclonal rabbit anti-osteocalcin (OC) antibodies were obtained from Abcam (Cambridge, UK).

Cell culture. HPLCs were obtained from the mid-roots of periodontally healthy permanent premolars and the third molar teeth extracted from 6 patients (age, 18-22 years), when informed consent had been signed. The study was approved by the Ethics Committee of Capital Medical University, School of Stomatology, Beijing, China. Briefly, the periodontal ligaments were minced, dispersed and incubated with DMEM containing $15 \%$ (v/v) FBS supplemented with antibiotics $(100 \mathrm{U} / \mathrm{ml}$ penicillin and $100 \mu \mathrm{g} / \mathrm{ml}$ streptomycin). All cells were cultured at $37^{\circ} \mathrm{C}$ in a humidified $5 \% \mathrm{CO}_{2}$ atmosphere. When a confluent monolayer was achieved, cells were harvested with $0.05 \%$ trypsin and $0.02 \%$ EDTA, transferred to a $25 \mathrm{~cm}^{2}$ diameter plastic culture bottle (Corning Inc., Corningy, NY, USA) and subcultured at a 1:2 split ratio with the initial confluent monolayer designated as one population doubling level. HPLCs at the third passage were used for the experiments described below.

Cell proliferation assay. The effect of icariin on cell proliferation was analyzed using an MTT assay. HPLCs were plated in flat-bottomed 96 -well plates $\left(2 \times 10^{3}\right.$ cells/well) in advance. Following incubation for $24 \mathrm{~h}$, the culture medium was replaced with fresh 5\% FBS-DMEM medium containing icariin $(0.001,0.01,0.1$ and $1 \mu \mathrm{g} / \mathrm{ml})$. The cultures were incubated for 2, 4 or 6 days. Cells treated with medium alone were used as a negative control and wells with medium alone were used as a blank control. Four hours prior to the end of the incubation, the cells were washed twice with phosphate-buffered saline ( $\mathrm{pH}$ 7.2) and incubated with $5 \mathrm{mg} / \mathrm{ml}$ MTT (20 $\mu \mathrm{l})$ for the last $4 \mathrm{~h}$. The medium was then decanted, formazan salts were dissolved in $200 \mu \mathrm{l}$ DMSO and the absorbance was determined at $490 \mathrm{~nm}$ using a high-throughput microplate spectrophotometer SpectraMax Plus ${ }^{384}$ (Molecular Devices, Sunnyvale, CA, USA).

qPCR. HPLCs were seeded on a $25 \mathrm{~cm}^{2}$ culture bottle at a density of $5 \times 10^{3}$ cells $/ \mathrm{cm}^{2}$. The RANKL, OPG, Cbfa1 and OC mRNA expression levels in the cells was analyzed 4 days following treatment with icariin $(0.001,0.01,0.1$ and $1 \mu \mathrm{g} / \mathrm{ml} \mu \mathrm{g} / \mathrm{ml}$ ) by qPCR. Cells treated with medium alone were considered as control cells. Briefly, the total cellular RNA was isolated using a Total RNA Extraction kit (Sunbio, Beijing, China) according to the manufacturer's instructions. Complementary DNA (cDNA) was synthesized using total RNA primed with Oligo(dT)12-18 Primer as described in the SuperScript ${ }^{\mathrm{TM}}$ III Reverse Transcriptase kit (Invitrogen Life Sciences, Carlsbad, CA, USA). The cDNA specimens were analyzed using SYBR-Green qPCR. Definitive primers are listed in Table I. qPCR was conducted using an ABI Prism 7700 system (Applied Biosystems, Carlsbad, CA, USA) under the following conditions: $2 \mathrm{~min}$ at $95^{\circ} \mathrm{C}, 45$ cycles of $20 \mathrm{sec}$ at $95^{\circ} \mathrm{C}, 25 \mathrm{sec}$ at $58^{\circ} \mathrm{C}$ and $30 \mathrm{sec}$ at $72^{\circ} \mathrm{C}$. Following PCR, a dissociation curve (melting curve) was constructed in the range of 65 to $95^{\circ} \mathrm{C}$.

Western blot analysis. Untreated control and treated HPLCs were rinsed with phosphate-buffered saline and solubilized with $1 \mathrm{X}$ radio immunoprecipitation assay buffer. The protein concentrations of RANKL, OPG, Cbfa1 and OC were determined using a Micro bicinchoninic acid (BCA) Protein Assay Reagent kit (Pierce Biotechnology Inc., Rockford, IL, USA). Equalized protein concentrations from each lysate were combined with $5 \mathrm{X}$ sodium lauryl sulfate (SDS) sample buffer and electrophoresed on a $10 \%$ SDS polyacrylamide gel and transferred to a polyvinylidene fluoride transfer membrane. The membrane was blocked with $5 \%$ non-fat milk in Tris buffer solution containing $0.05 \%$ Tween-20 (TBST) for $1 \mathrm{~h}$. The membranes were incubated overnight with the appropriate dilutions of primary antibodies in blocking buffer at $4^{\circ} \mathrm{C}$. The following day, the membranes were washed and incubated for 2-3 $\mathrm{h}$ with alkaline phosphatase-conjugated secondary antibody solution in blocking buffer and washed three times with TBST. The proteins were visualized by 
enhanced chemiluminescence according to the manufacturer's instructions (Amersham Biosciences, Piscataway, NJ, USA). Western blot analysis results were quantified by densitometry (Software Labworks 4.6; UVP, Cambridge, UK).

Statistical analysis. All values were presented as the mean \pm standard deviation. Statistical analysis was conducted using one-way analysis of variance and Wilcoxon's signed-rank test. $\mathrm{P}<0.05$ was considered to indicate a statistically significant difference.

\section{Results}

Cell proliferation. The effect of icariin $(0.001,0.01,0.1$ and $1 \mu \mathrm{g} / \mathrm{ml}$ ) on the proliferation of HPLCs was analyzed. Fig. 1 shows that compared with the control, icariin promoted cell proliferation in a dose- and time-dependent manner. The highest stimulatory activity was observed with an icariin concentration of $0.01 \mu \mathrm{g} / \mathrm{ml}$ at 4 days and declined thereafter.

Effect of icariin on RANKL and OPG mRNA and protein expression in HPLCs. The mRNA levels of RANKL and OPG expressed by HPLCs exposed to icariin at 4 days were analyzed by qPCR. $\beta$-actin was used as a reference gene. Fig. 2 shows that HPLCs expressed RANKL and OPG genes under physiological conditions. Low-dose icariin with a concentration of $0.001 \mu \mathrm{g} / \mathrm{ml}$ did not exhibit a significant effect on RANKL and OPG expression in HPLCs. However, at a concentration of $0.01 \mu \mathrm{g} / \mathrm{ml}$, icariin significantly increased OPG while decreasing RANKL expression and resulted in the lowest RANKL/OPG expression ratio compared with the control and other treatment groups. At concentrations of 0.1 and $1 \mu \mathrm{g} / \mathrm{ml}$, treatment with icariin resulted in a decrease of OPG expression, an increase in RANKL expression and a higher RANKL/OPG expression ratio.

Western blot analysis was conducted to analyze RANKL and OPG protein production. HPLCs expressed RANKL and OPG under physiological conditions. Icariin exhibited a dose-dependent effect on decreasing RANKL and increasing OPG production in HPLCs. This was greatest in the $0.01 \mu \mathrm{g} / \mathrm{ml}$ icariin group, which exhibited the lowest RANKL/OPG production ratio (Fig. 3).

Effect of icariin on Cbfal and OC $m R N A$ and protein expression in HPLCs. To investigate the effects of icariin on the regulation of osteogenic differentiation of periodontal ligament cells, Cbfal and OC expression in HPLCs was analyzed. $\beta$-actin was used in the same samples as a reference gene. HPLCs expressed Cbfa1 and OC under physiological conditions. Icariin exhibited a dose-dependent effect on the induction of Cbfa1 and OC mRNA expression in HPLCs. Cbfa1 and OC expression was induced in the $0.001 \mu \mathrm{g} / \mathrm{ml}$ icariin group. The highest expression value was observed in the $0.01 \mu \mathrm{g} / \mathrm{ml}$ icariin group. At concentrations of 0.1 and $1 \mu \mathrm{g} / \mathrm{ml}$, treatment with icariin resulted in the decrease in Cbfa1 and OC expression (Fig. 4).

In addition, icariin also exhibited a dose-dependent effect on the induction of $\mathrm{Cbfa} 1$ and OC protein production in HPLCs. The most marked effect was observed in the $0.01 \mu \mathrm{g} / \mathrm{ml}$ icariin group (Fig. 5).

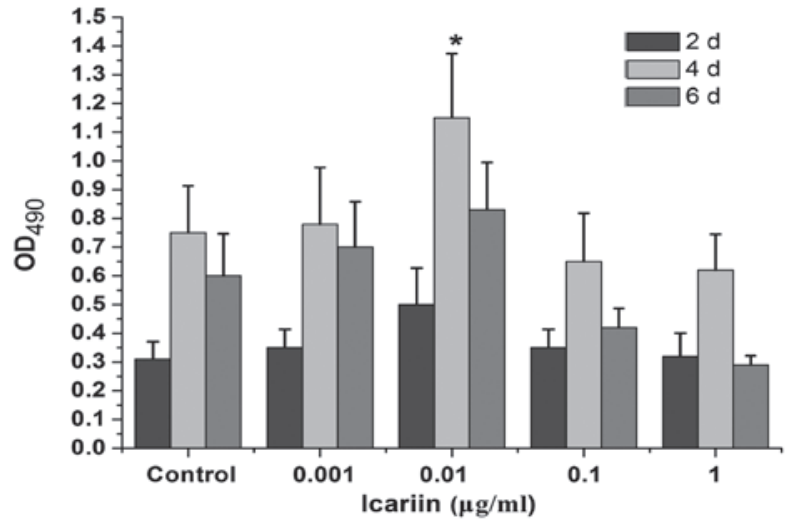

Figure 1. Effect of icariin $(0.001,0.01,0.1$ and $1 \mu \mathrm{g} / \mathrm{ml})$ on the proliferation of HPLCs at 2, 4 and 6 days. The cell viability in the presence of icariin increased in a dose- and time-dependent manner, and the maximum stimulatory effect on cell proliferation was achieved on day 4 with $0.01 \mu \mathrm{g} / \mathrm{ml}$ icariin. Data are presented as the mean \pm SD $(n=12)$. ${ }^{*} \mathrm{P}<0.05$ compared with the control group. $\mathrm{OD}_{490}$, optical density; HPLCs, human periodontal ligament cells.

A

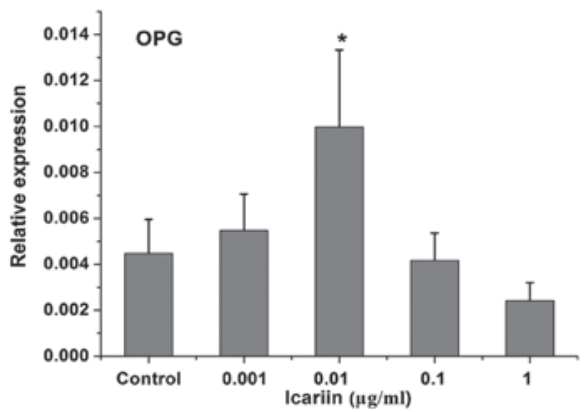

B



C

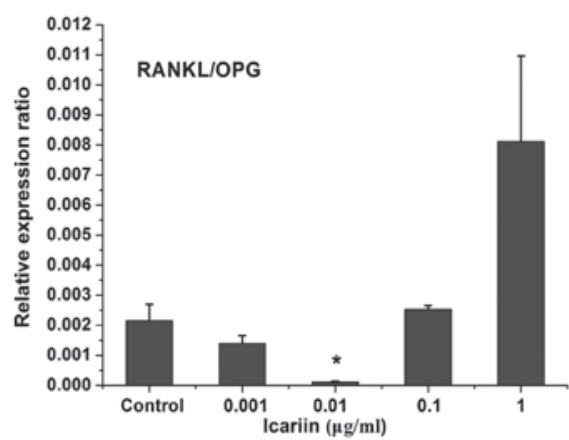

Figure 2. Regulation of (A) OPG, (B) RANKL gene expression and (C) RANKL/OPG expression ratio in HPLCs treated with different concentrations of icariin for 4 days. The mRNA expression levels were measured by qPCR analysis and normalized against the expression levels of $\beta$-actin (internal control). Data are presented as the mean $\pm \mathrm{SD}(\mathrm{n}=3) .{ }^{*} \mathrm{P}<0.05$ compared with the control group. OPG, osteoprotegerin; RANKL, receptor activator of nuclear factor- $\kappa \mathrm{B}$ ligand; HPLCs, human periodontal ligament cells. 

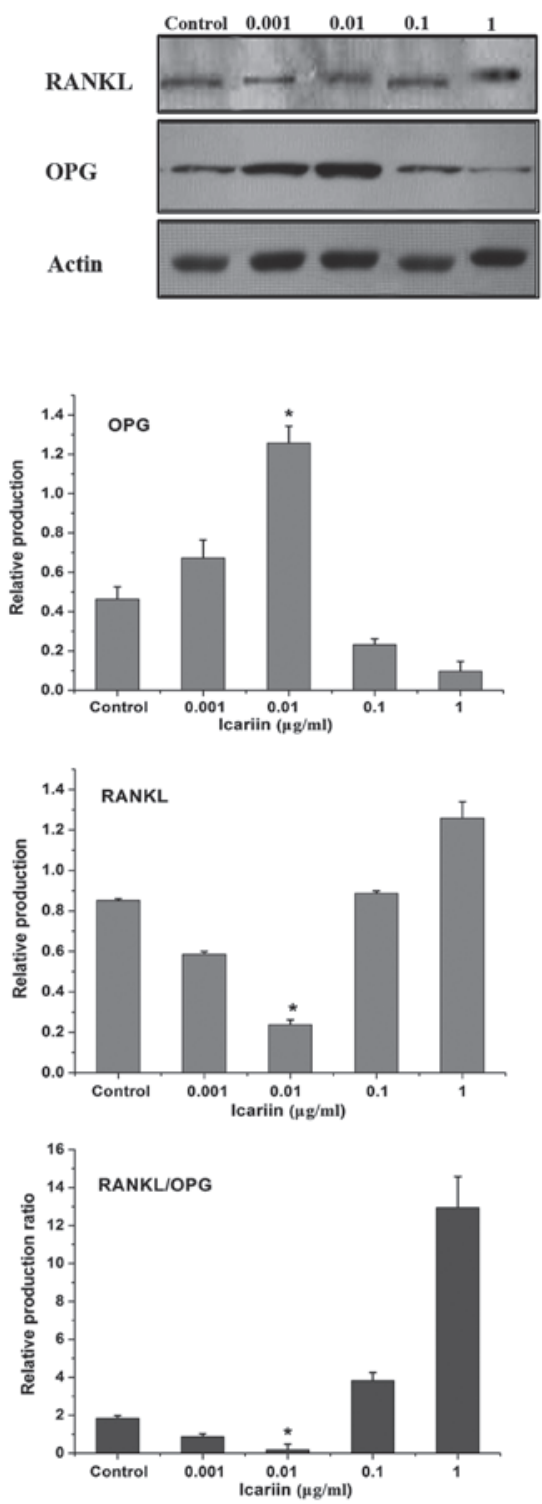

Figure 3. Regulation of OPG and RANKL protein production in HPLCs treated with different concentrations of icariin for 4 days. The protein production levels of OPG and RANKL were measured by western blot analysis, and normalized against the expression levels of $\beta$-actin (internal control). (A) Representative results of one of the triplicate experiments are shown. (B) OPG protein production levels. (C) RANKL protein production levels; (D) RANKL/OPG production ratio. Data are presented as the mean $\pm \mathrm{SD}$ $(n=3) .{ }^{*} \mathrm{P}<0.05$ compared with the control group. OPG, osteoprotegerin RANKL, receptor activator of nuclear factor- $\mathrm{kB}$ ligand; HPLCs, human periodontal ligament cells.

\section{Discussion}

Previous studies have demonstrated that icariin stimulates bone marrow stromal cells (MSCs), osteoblast proliferation and osteogenic differentiation in a time- and dose-dependent manner $(9,16,17)$. The results of the present study were in concordance with previous studies and demonstrated that icariin enhanced human periodontal ligament cell proliferation. In the present study, treatment with $0.01 \mu \mathrm{g} / \mathrm{ml}$ icariin at 4 days showed the greatest stimulatory effect on HPLCs proliferation. This suggested icariin may exhibit an osteogenic function by stimulating periodontal ligament cell proliferation. Compared with previous reports, the differences in the
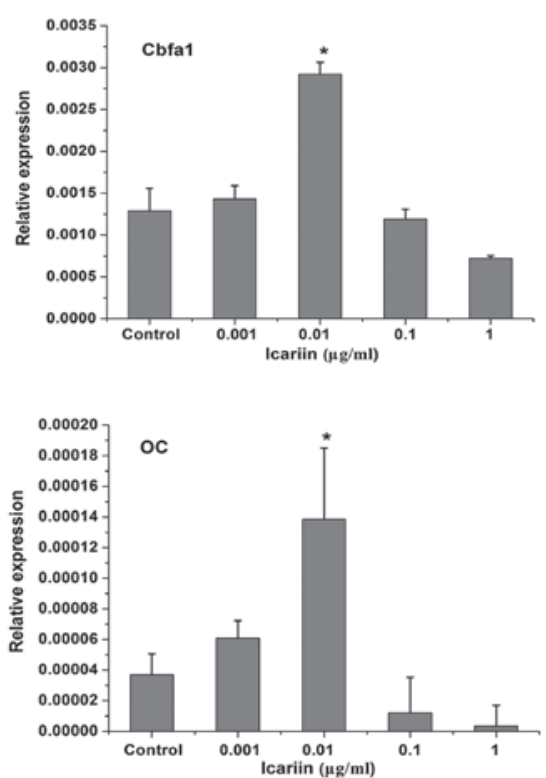

Figure 4. Regulation of (A) Cbfa1 and (B) OC gene expression in HPLCs treated with different concentrations of icariin for 4 days. The mRNA expression levels were measured by qPCR analysis and normalized against the expression levels of $\beta$-actin (internal control). Data are presented as the mean $\pm \mathrm{SD}(\mathrm{n}=3) .{ }^{*} \mathrm{P}<0.05$, compared with the control group. Cbfa1, core binding factor $\alpha 1$; OC, osteocalcin; HPLCs, human periodontal ligament cells.
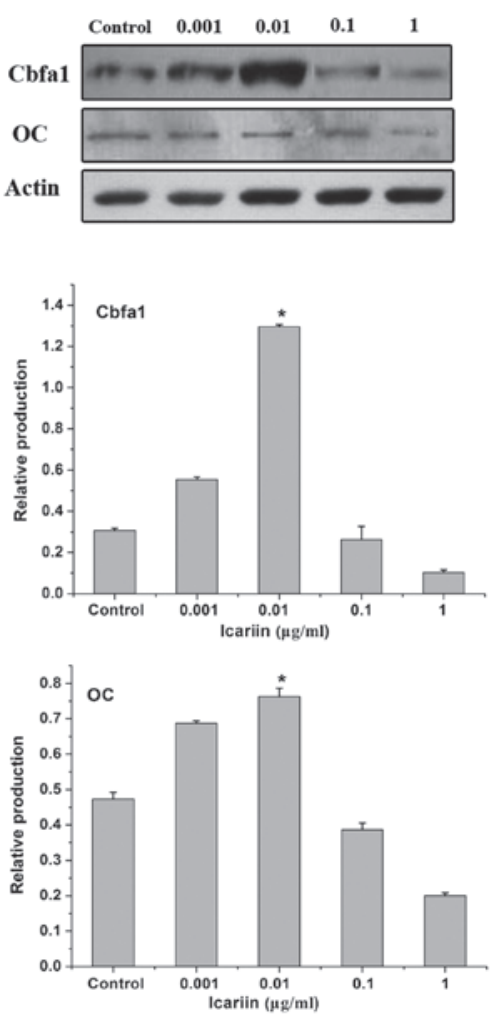

Figure 5. Regulation of Cbfa1 and OC protein production in HPLCs treated with different concentrations of icariin for 4 days. The protein production levels of $\mathrm{Cbfa} 1$ and $\mathrm{OC}$ were measured by western blot analysis and normalized against the expression levels of $\beta$-actin (internal control). (A) Representative results of the triplicate experiments are shown. (B) Cbfa1 protein production levels. (C) OC protein production levels. Data are presented as the mean $\pm \mathrm{SD}(\mathrm{n}=3) .{ }^{*} \mathrm{P}<0.05$ compared with the control group. Cbfa1, core binding factor $\alpha 1$; OC, osteocalcin; HPLCs, human periodontal ligament cells. 
effects of the icariin concentration may be due to cell type, inoculation number and culture conditions.

It was determined that icariin promoted HPLC proliferation, and thus, the next aim was to investigate the regulation of certain biomarkers involved in alveolar bone resorption or formation. Numerous studies have demonstrated that RANKL and its decoy receptor, OPG, were essential regulatory molecules in osteoclastogenesis and bone resorption, in physiological and pathological conditions. Significantly higher levels of RANKL and lower osteoprotegerin protein levels are expressed in the periodontitis tissue. The RANKL/OPG ratio in the gingival crevice fluid was significantly increased in periodontal disease patients compared with healthy subjects (18). Osteoblasts as well as other cells, such as periodontal ligament cells participated in the regulation of RANKL and OPG in periodontal tissue. OPG synthesized locally by periodontal ligament cells regulated the resorption of alveolar bone via cytokines, such as interleukin-1 $\beta$ and tumor necrosis factor- $\alpha$ (19). Inactivation of OPG is involved in osteoclast formation by periodontal ligament fibroblasts (20). Icariin was shown to be a regulatory agent in the upregulation of OPG and downregulation of RANKL gene expression in osteoblasts and UMR 106 cells (13). In the present study, icariin at a concentration of $0.01 \mu \mathrm{g} / \mathrm{ml}$ significantly increased OPG while decreasing RANKL mRNA expression and protein production, and obtained the lowest RANKL/OPG expression ratio. However, a high-dose icariin $(0.1 \mu \mathrm{g} / \mathrm{ml}$ and $1 \mu \mathrm{g} / \mathrm{ml})$ resulted in an increased RANKL/OPG expression ratio, possibly due to cell cytotoxicity. A previous study by Fan et al, demonstrated that icariin exhibited a dose-dependent effect on the proliferation and osteogenic differentiation of human bone mesenchymal stem cells at a suitable concentration range; however, when the concentration was $>10^{-5} \mathrm{M}$, cytotoxicity limited its effect (9). It was suggested that icariin may inhibit osteoclast differentiation and alveolar bone resorption by regulating RANKL and OPG levels in periodontal ligament cells.

Cbfa1 is an essential transcription factor in osteoblast differentiation (21-23). Mesenchymal stem cells transfected with an adenovirus encoding Cbfal resulted in the increase of a number of osteoblastic markers including osteocalcin, osteopontin and type I collagen expression, and also induced a significantly increased bone formation and marrow cavity rebuilding (21). OC is a small, acidic extracellular protein synthesized by osteoblasts during bone formation (24). Bone cell activity is indirectly evaluated by the measurement of specific biochemical markers of bone formation such as osteocalcin; the greater the OC expression the more bone formed (25). Previous studies demonstrated that icariin increased the proliferation of osteoblasts and the gene expression of Cbfa1/Runx 2 in these cells. In addition, it also increased OPG gene expression but downregulated RANKL gene expression (26). In the present study, similar effects of icariin were observed and certain concentrations markedly increased Cbfa1 and OC mRNA expression, and protein production in periodontal ligament cells. The results suggested that icariin may be considered as a growth factor similar to bone morphogenetic protein-2 and may be used to promote bone formation and periodontal regeneration.

In conclusion, the results demonstrated the potential use of icariin in treating alveolar bone resorption and promoting peri- odontal tissue regeneration in periodontitis, due to its ability to stimulate the proliferation and osteogenic differentiation of human periodontal ligament cells and inhibition of osteoclast differentiation. However, the present study only determined the effects in vitro, and thus, the effect of icariin in periodontal tissue regeneration in vivo requires further investigation.

\section{Acknowledgements}

This study was supported by the financial support of Beijing Natural Science Foundations (grant no. 7122077) and the Beijing Municipal Health Bureau (grant no. JJ2009-26), China.

\section{References}

1. Dikbas I, Tanalp J, Tomruk CO and Koksal T. Evaluation of reasons for extraction of crowned teeth: a prospective study at a university clinic. Acta Odontol Scand 71: 848-856, 2013.

2. Kobayashi Y, Udagawa N and Takahashi N: Action of RANKL and OPG for osteoclastogenesis. Crit Rev Eukaryot Gene Expr 19: 61-72, 2009.

3. Boyce BF and Xing L: Functions of RANKL/RANK/OPG in bone modeling and remodeling. Arch Biochem Biophys 473: 139-146, 2008.

4. Seo BM, Miura M, Gronthos S, et al: Investigation of multipotent postnatal stem cells from human periodontal ligament. Lancet 364: 149-155, 2004.

5. Gronthos S, Mrozik K, Shi S and Bartold PM: Ovine periodontal ligament stem cells: isolation, characterization, and differentiation potential. Calcif Tissue Int 79: 310-317, 2006.

6. Wada N, Maeda $\mathrm{H}$, Tanabe $\mathrm{K}$, et al: Periodontal ligament cells secrete the factor that inhibits osteoclastic differentiation and function: the factor is osteoprotegerin/osteoclastogenesis inhibitory factor. J Periodontal Res 36: 56-63, 2001.

7. Hasegawa T, Yoshimura Y, Kikuiri T, et al: Expression of receptor activator of NF-kappa $\mathrm{B}$ ligand and osteoprotegerin in culture of human periodontal ligament cells. J Periodontal Res 37: 405-411, 2002.

8. Kanzaki H, Chiba M, Shimizu Y and Mitani H: Dual regulation of osteoclast differentiation by periodontal ligament cells through RANKL stimulation and OPG inhibition. J Dent Res 80: 887-891, 2001.

9. Fan JJ, Cao LG, Wu T, et al: The dose-effect of icariin on the proliferation and osteogenic differentiation of human bone mesenchymal stem cells. Molecules 16: 10123-10133, 2011.

10. Hsieh TP, Sheu SY, Sun JS and Chen MH: Icariin inhibits osteoclast differentiation and bone resorption by suppression of MAPKs/NF- $x$ B regulated HIF-1 $\alpha$ and PGE(2) synthesis. Phytomedicine 18: 176-185, 2011.

11. Ma HP, Ming LG, Ge BF, et al: Icariin is more potent than genistein in promoting osteoblast differentiation and mineralization in vitro. J Cell Biochem 112: 916-923, 2011.

12. Cao H, Ke Y, Zhang Y, Zhang CJ, Qian W and Zhang GL: Icariin stimulates MC3T3-E1 cell proliferation and differentiation through up-regulation of bone morphogenetic protein-2. Int J Mol Med 29: 435-439, 2012.

13. Mok SK, Chen WF, Lai WP, et al: Icariin protects against bone loss induced by oestrogen deficiency and activates oestrogen receptor-dependent osteoblastic functions in UMR 106 cells. Br J Pharmacol 159: 939-949, 2010.

14. Nian H, Ma MH, Nian SS and Xu LL: Antiosteoporotic activity of icariin in ovariectomized rats. Phytomedicine 16: 320-326, 2009.

15. Zhao J, Ohba S, Komiyama Y, Shinkai M, Chung UI and Nagamune T: Icariin: a potential osteoinductive compound for bone tissue engineering. Tissue Eng Part A 16: 233-243, 2010.

16. Chen KM, Ge BF, Ma HP, Liu XY, Bai MH and Wang Y: Icariin, a flavonoid from the herb Epimedium enhances the osteogenic differentiation of rat primary bone marrow stromal cells. Pharmazie 60: 939-942, 2005.

17. Yin XX, Chen ZQ, Liu ZJ, Ma QJ and Dang GT: Icariine stimulates proliferation and differentiation of human osteoblasts by increasing production of bone morphogenetic protein 2 . Chin Med J (Engl) 120: 204-210, 2007. 
18. Mogi M, Otogoto J, Ota N and Togari A: Differential expression of RANKL and osteoprotegerin in gingival crevicular fluid of patients with periodontitis. J Dent Res 83: 166-169, 2004.

19. Sakata M, Shiba H, Komatsuzawa H, et al: Expression of osteoprotegerin (osteoclastogenesis inhibitory factor) in cultures of human dental mesenchymal cells and epithelial cells. J Bone Miner Res 14, 1486-1492, 1999.

20. Hasegawa T, Yoshimura Y, Kikuiri T, et al: Expression of receptor activator of NF-kappa B ligand and osteoprotegerin in culture of human periodontal ligament cells. J Periodontal Res 37: 405-411, 2002.

21. Dong SW, Ying DJ, Duan XJ, et al: Bone regeneration using an acellular extracellular matrix and bone marrow mesenchyma stem cells expressing Cbfa1. Biosci Biotechnol Biochem 73: 2226-2233, 2009.
22. Ducy P: Cbfa1: a molecular switch in osteoblast biology. Dev Dyn 219: 461-471, 2000.

23. Lian JB, Stein JL, Stein GS, et al: Runx2/Cbfa1 functions: diverse regulation of gene transcription by chromatin remodeling and co-regulatory protein interactions. Connect Tissue Res 44 Suppl 1: 141-148, 2003.

24. Patterson-Buckendahl P: Osteocalcin is a stress-responsive neuropeptide. Endocr Regul 45: 99-110, 2011.

25. Maïmoun L, Fattal C and Sultan C: Bone remodeling and calcium homeostasis in patients with spinal cord injury: a review. Metabolism 60: 1655-1663, 2011.

26. Hsieh TP, Sheu SY, Sun JS, Chen MH and Liu MH: Icariin isolated from Epimedium pubescens regulates osteoblasts anabolism through BMP-2, SMAD4, and Cbfa1 expression. Phytomedicine 17: 414-423, 2010. 